\title{
Investigating recording patterns using magnetic force microscopy
}

\author{
J.P.J. (Hans) Groenland ${ }^{\text {a,1 }}$, G.J.P. (Peter) van Engelen ${ }^{\text {b }}$, Jan P.C. Bernards ${ }^{\text {b }}$ \\ and Hugo A.J. Cramer ${ }^{b}$ \\ ${ }^{a}$ University of Twente, Faculty of Electrical Engineering (MESA Research Institute), P.O. Box 217, NL-7500 AE Enschede, \\ The Netherlands \\ ${ }^{b}$ Philips Research Laboratories Eindhoven, P.O. Box 80.000, NL-5600 JA Eindhoven, The Netherlands
}

\begin{abstract}
The magnetic force microscope is a valuable tool for the qualitative analysis of local phenomena in magnetic recording media. To study the possibilities for a more quantitative analysis, the MFM results have been compared with recording results. It is shown that such analysis is hampered by the nonlinear transfer characteristic and by the masking of local high-definition details due to the application of an auxiliary electrostatic field in the current instrument.
\end{abstract}

\section{Introduction}

The magnetic force microscope (MFM) is appropriate for mapping the stray magnetic fields of a sample by scanning over its surface with a magnetic tip. The MFM is a variant of the atomic force microscope [1] and has created a furore in recent years. Special features of the instrument are its high sensitivity, spatial resolution and the ease of sample preparation.

Several papers have described the MFM as an instrument for the qualitative analysis of magnetic structures (for example refs. [2-4]). In fig. 1 a typical result obtained with a dynamic MFM is reproduced. By observing the figure, it may be suggested that it represents the local magnetic field. However, the correctness of such an interpretation is not self-evident. Another important issue is the attainable lateral resolution of the MFM.

In this paper we investigate the possibilities of applying the MFM for the quantitative analysis of recording patterns in a magnetic medium. Questions addressed include: the possibility to interpret the instrument's quantitative output, and the extra value of the MFM technique with respect to measurements with an inductive ring head in a magnetic recording setup. In view of these items tape recordings with various write currents and wavelengths have been played back with

Correspondence to: Dr. J.P.J. Groenland, University of Twente, Faculty of Electrical Engineering (MESA Research Institute), P.O. Box 217, NL-7500 AE Enschede, The Netherlands. Tel.: +31-53-892713; telefax: +31-53-309547; telex: 44200 thtes nl.

1 This work was carried out during sabbatical leave at Philips Research Laboratories, Eindhoven, The Netherlands. an inductive ring head and the results are compared with the output of the MFM of the same recording.

\section{Setup and description of the MFM}

A magnetic force microscope has been applied for the two-dimensional mapping of magnetic field structures at the surface of a flat substrate. Magnetic tips are produced by depositing a thin layer of cobalt on an etched tungsten wire tip [5]. This wire forms a cantilever and the tip performs a small oscillating movement in the stray field of the substrate.

The instrument used is similar to the setup described by Den Boef [6,7] and operates in the 'constant-gradient' mode. This means that during the scanning operation the distance between the sample and tip is constantly adjusted in such a way that the first force derivative to the distance is held at a fixed value. This can be realized by exciting the cantilever close to its resonance frequency with an electrostatic ac field and by measuring the phase of the forced vibration. The force gradient changes the effective spring constant and thus the resonance frequency of the cantilever. The distance at which the force gradient is constant is the output of the instrument.

The introduction of an (attractive) electrostatic dc field caused by a voltage $U_{\mathrm{b}}(2-10 \mathrm{~V})$ between tip and sample actually allows for the control system to be stable $[3,7]$. The electrostatic force gradient increases with decreasing tip-sample distance $z_{0}$ by a factor $U_{\mathrm{b}} / z_{0}$ [7]. The combined effects of magnetic and electrostatic force gradients result in an effective force gradient with a constant sign. Therefore, the distance between tip and sample never tends to 'become negative', i.e. crashing of the tip is averted. An undesirable effect of the electrostatic force is the pronounced non- 


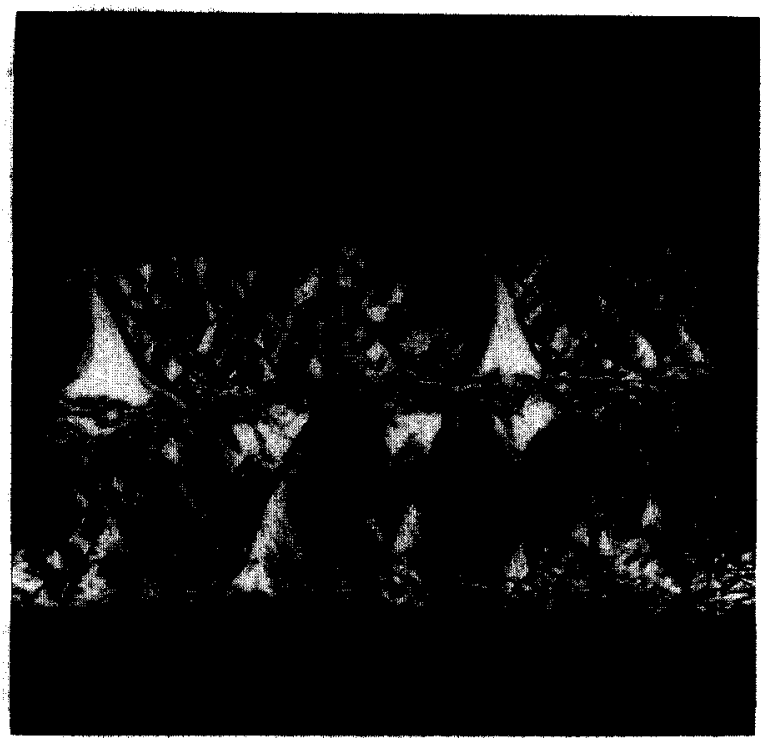

Fig. 1. Perspective image, representing the output signal (after the application of an image-processing step) of a dynamic MFM after scanning an ME tape surface. Tracks $5 \mu \mathrm{m}$ wide have been recorded with a random bit pattern with bit lengths of $0.25 \mu \mathrm{m}$.

linear behaviour of the MFM, observed as an asymmetrical imaging response to magnetic force derivatives of opposite sign. A more linear transfer characteristic may only be expected for small variations in the MFM signals.

\section{Quantitative analysis of MFM measurement results}

In a recording setup, magnetic tracks in metalevaporated (ME) tape samples were recorded and played back. Samples with recording tracks with wavelengths from 0.5 to $20 \mu \mathrm{m}$ were studied by MFM. The MFM image in fig. 2 shows two overlapping magnetic tracks: a $\lambda=1 \mu \mathrm{m}$ track is partly overwritten by a $\lambda=12 \mu \mathrm{m}$ track. Indicated in this figure are the positions of three particular scans, which are graphically reproduced in fig. 3 . These scans are located in the $\lambda=1 \mu \mathrm{m}$ track, the intermediate region $1 \mu \mathrm{m} / 12 \mu \mathrm{m}$ and the $\lambda=12 \mu \mathrm{m}$ track, respectively.

\subsection{Resolution}

When discussing the resolution of an imaging method, we focus on the smallest discernible details. For the MFM, a resolution of $50 \mathrm{~nm}$ has been obtained. In our case, the bits of $0.25 \mu \mathrm{m}$ size in the image of fig. 1 are easily resolved.

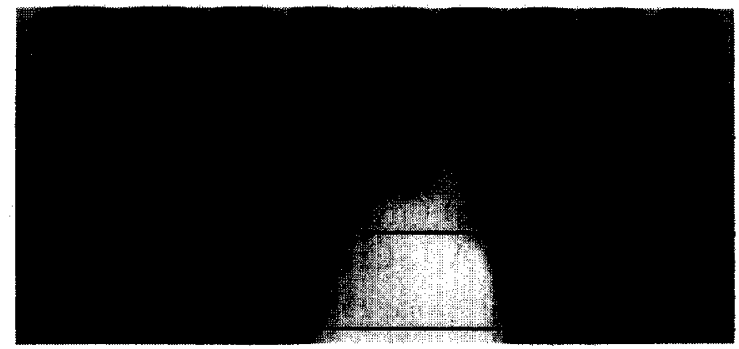

Fig. 2. Two-dimensional plot of the MFM output (after application of an image-processing step) for two overlapping magnetic tracks. The height parameter is indicated by means of a grey gradation ('white' reproducing the maximum separation between tip and sample).

The main problem encountered was the difference in sensitivity for long- and short-wavelength signals. This phenomenon is expressed by the results given in fig. 4, where the signal amplitudes of inductive-head and MFM measurements are given as a function of the inverse wavelength. Both curves show a roll-off behaviour, but the cut-off frequency appears to be lower for the MFM signal $(\lambda=5 \mu \mathrm{m})$ than for the ring head $(\lambda=2 \mu \mathrm{m})$. For the MFM, the roll-off curves for both scanning directions are similar. A particular result of this phenomenon is clearly visible in figs. 2 and 3 .
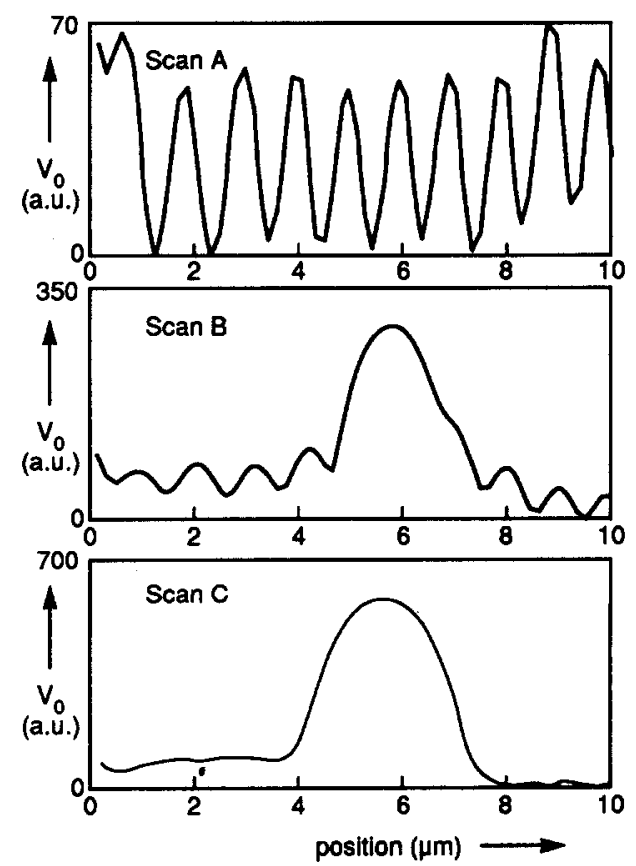

Fig. 3. Quantitative representation of the MFM output for three scans as indicated in fig. 2. Note the difference in vertical scales. 


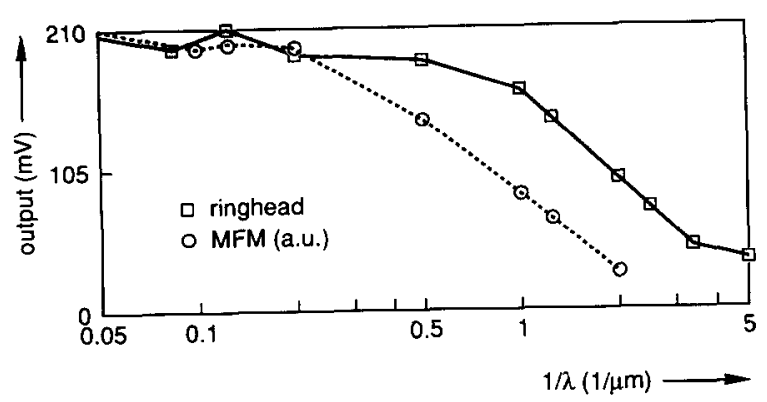

Fig. 4. Amplitudes of pulses measured by inductive ring head and MFM, respectively, for a range of wavelength values.

Based on the physics of the recording process, a reasonably straight boundary between both tracks has to be expected. However, the image in fig. 2 as well as the quantitative MFM output signal (scan B in fig. 3) shows the signal of the $12 \mu \mathrm{m}$ reversal overlapping the signals of the first written track by about $1.7 \mu \mathrm{m}$. This is in agreement with the relatively large pulse-widthat-half-height of $3 \mu \mathrm{m}$, observed in scan $\mathrm{C}$ of the $\lambda=12 \mu \mathrm{m}$ track.

For a linear measuring system the problem of a high value of the cut-off wavelength can be solved by appropriate filtering. However, in the next section we show the current system behaves essentially nonlinearly.

\subsection{Linearity}

Because the resolution is dependent on the scanning height [4], there are signal-dependent local variations in the resolution. This can be seen in scan B, where at the position of the isolated magnetic reversal in the $\lambda=12 \mu \mathrm{m}$ signal, the tip reaches a relatively large flying height of the order of $1 \mu \mathrm{m}$, resulting in a suppression of the signal of the $\lambda=1 \mu \mathrm{m}$ reversals.

The electrostatic field is the second reason why the MFM output is not linearly dependent on the perpendicular field component of the medium stray field. This is observed in the $\lambda=12 \mu \mathrm{m}$ curve of fig. 3 (scan C). The signals with a negative polarity are strongly reduced in amplitude compared to positive signals. In order to study the correspondence between the signals of the inductive read head and the MFM in more detail, patterns were recorded with various write currents and played back. A wavelength of $10 \mu \mathrm{m}$ was chosen, resulting in 'isolated' read pulses. The output signals of both systems appeared to increase monotonously in the write-current range of 2-8 $\mathrm{mA}$. As expected, only low-amplitude magnetization patterns (write currents $<3 \mathrm{~mA}$ ) were observed to produce (nearly) symmetric MFM output signals.

\section{Conclusions and discussion}

The MFM is well suited for the investigation of magnetic phenomena at the surface of a limited area of the medium with a high resolution in both directions. In order to interpret the measurement results, knowledge of the instrument's characteristics is indispensable. For quantitative analysis, the mechanical and magnetic characteristics of the tips have to be stable and preferably reproducible.

It has been shown that the application of an electrostatic field introduces nonlinearities, which in our experiments were quite pronounced. However, the electrostatic field also offers clear advantages. Detection of stray fields with alternating polarity is possible, the tip-sample distance is easily adjusted with the help of the applied electrical voltage, and the probability of crashes between tip and sample is greatly reduced.

A more linear characteristic of the same instrument would be obtained using tips with smaller magnetic moments. However, in that case the signals are smaller and higher demands are made upon the stability of the setup. Another consequence is the decreased resistance against slight crashes.

A second observation is the high value of the cut-off wavelength in the roll-off plot. As shown, this hinders the detection of details in the presence of long-wavelength signal and noise. It can also easily lead to misinterpretations of side-writing effects. Linear filtering is prohibited by the nonlinearities mentioned above, and by the variation in resolution due to the variable flying height. Smaller tips and lower flying heights can lead to a smaller cut-off frequency, but increase the risk of crashing.

A possible way to solve both problems is to change the measuring procedure, e.g. by scanning the surface a second time at constant height and measuring the phase variations. Only then is it possible to apply analysis methods like Fourier transform and data processing in the frequency domain.

\section{References}

[1] Y. Martin, D. Rugar and H.K. Wickramasinghe, Appl. Phys. Lett. 52 (1988) 244.

[2] C. Schönenberger and S.F. Alvarado, Z. Phys. B80 (1990) 373.

[3] D. Rugar, H.M. Mamin, P. Guethner, S.E. Lambert, J.E. Stern, I. McFadyen and T. Yogi, J. Appl. Phys. 68 (1990) 1169.

[4] J.P.C. Bernards and A.J. den Boef, IEEE Trans. Magn. MAG-26 (1990) 1515.

[5] A.J. den Boef, Appl. Phys. Lett. 56 (1990) 2045.

[6] A.J. den Boef, Appl. Phys. Lett. 55 (1989) 439.

[7] A.J. den Boef, PhD Thesis, University of Twenty (1991). 